\title{
An endovascular assisted, nonocclusive cerebral bypass: a technical feasibility study in a rabbit model
}

\author{
${ }^{*}$ Alexander Spiessberger, MD,,2 Fabio Strange, MD,,2 Basil Erwin Gruter, MD,1,2 \\ Stefan Wanderer, MD, ${ }^{1,2}$ Daniela Casoni, DVM, PhD, ${ }^{3}$ Philipp Gruber, MD, ${ }^{4}$ Michael Diepers, MD, ${ }^{4}$ \\ Luca Remonda, MD, ${ }^{4}$ Javier Fandino, MD, ${ }^{1,2}$ Javier Añon, MD, ${ }^{4}$ and Serge Marbacher, MD, PhD ${ }^{1,2}$

\begin{abstract}
${ }^{1}$ Department of Neurosurgery, Kantonsspital Aarau; ${ }^{2}$ Department of Biomedical Research, Cerebrovascular Research Group, University of Bern; ${ }^{3}$ Department of Biomedical Research, Faculty of Medicine, University of Bern; and ${ }^{4}$ Division of Neuroradiology, Kantonsspital Aarau, Switzerland
\end{abstract}

\begin{abstract}
OBJECTIVE Temporary parent vessel occlusion performed to establish a high-flow interpositional bypass carries the risk of infarcts. The authors investigated the feasibility of a novel technique to establish a high-flow bypass without temporary parent vessel occlusion in order to lower the risk of ischemic complications.
\end{abstract}

METHODS In 10 New Zealand white rabbits, a carotid artery side-to-end anastomosis was performed under parent artery patency with a novel endovascular balloon device. Intraoperative angiography, postoperative neurological assessments, and postoperative MRI/MRA were performed to evaluate the feasibility and safety of the novel technique.

RESULTS A patent anastomosis was established in 10 of 10 animals; 3 procedure-related complications occurred. No postoperative focal neurological deficits were observed. The MRI/MRA findings include no infarcts and bypass patency in $50 \%$ of the animals.

CONCLUSIONS The authors demonstrated the feasibility of an endovascular assisted, nonocclusive high-flow bypass. Future refinement of the device and technique in an animal model is necessary to lower the complication rate and increase patency rates.

https://thejns.org/doi/abs/10.3171/2020.3.JNS20334

KEYWORDS cerebral bypass; nonocclusive technique; bypass surgery; vascular disorders; New Zealand white rabbit

$\sim$ EREBRAL revascularization by means of an arterioarterial bypass can be indicated in the treatment of a subset of complex aneurysms that are not amenable to conventional surgical or endovascular treatment, in tumorous lesions with involvement of cerebral arteries, or in occlusive vascular diseases such as moyamoya or arteriosclerotic disease. ${ }^{1-3}$ Flow replacement of a proximal cerebral vessel, such as the terminal internal carotid artery (ICA), is ideally treated with a high-flow bypass, as opposed to low-flow bypass constructs such as Yaşargil's prototype bypass, superficial temporal artery- $\mathrm{M}_{4}$ segment of the middle cerebral artery (MCA). ${ }^{4}$ Typically, a highflow bypass uses a saphenous vein or radial artery graft, the external carotid artery is the proximal donor vessel, and the ICA or $\mathrm{MCA}^{5}$ distally is the recipient vessel. Other high-flow bypass constructs are the internal maxillary artery-MCA bypass and the petrous ICA-supraclinoid ICA (Fukushima) bypass. ${ }^{6}$

If the conventional technique to create a high-flow bypass is applied, the parent and recipient vessels must be temporarily clipped to perform the anastomoses. However, temporary clipping of the proximal cerebral vessels carries the risk of ischemic complications, particularly if occlusion times exceed certain limits, which were found to be $10-19$ minutes. ${ }^{78}$ In the more recent past an alternative technique using an endovascular laser (excimer laserassisted nonocclusive anastomosis [ELANA] technique) has been developed, which requires no parent vessel occlusion. ${ }^{1}$ We seek to develop yet another type of nonocclusive bypass, which uses an endovascular device, requiring

ABBREVIATIONS CA = carotid artery; $\mathrm{CCA}=$ common carotid artery; $\mathrm{DSA}=$ digital subtraction angiography; DWI = diffusion-weighted imaging; ENO = endovascular assisted nonocclusive; FA = femoral artery; ICA = internal carotid artery; MCA = middle cerebral artery; PCA = posterior cerebral artery; TOF-A = time-of-flight angiography. SUBMITTED February 3, 2020. ACCEPTED March 31, 2020.

INCLUDE WHEN CITING Published online June 5, 2020; DOI: 10.3171/2020.3.JNS20334.

${ }^{*}$ A.S. and F.S. contributed equally to this work. 
minimal parent and recipient vessel exposure. These features could be particularly suitable for posterior circulation bypasses and the Fukushima bypass. ${ }^{9}$

In a rabbit model we attempted to create a cerebral high-flow bypass by using an endovascular balloon device, which seals the vessel wall of the parent vessel, enabling the creation of a dry anastomosis while the central blood flow in the parent vessel is maintained (Fig. 1). No temporary clamping is necessary, thus minimizing the risk of ischemia, and only minimal parent vessel exposure is required. The technique is termed the endovascular assisted nonocclusive (ENO) bypass procedure.

\section{Methods}

Approval for this study was obtained from the local Committee for Animal Care. All animal experiments were conducted in accordance with Animal Research: Reporting In Vivo Experiments (ARRIVE) guidelines ${ }^{10}$ and with the Guide for the Care and Use of Laboratory Animals at our Experimental Surgical Facility. Certified veterinarians performed general anesthesia and postoperative care.

Ten female New Zealand white rabbits (Charles River), all 16-18 weeks of age and weighing 3-5 kg each, underwent an ENO bypass procedure consisting of right cervical carotid artery (CA)-to-left cervical CA transposition (end-to-side anastomosis) under general anesthesia. The diameter of the CA, according to previous anatomical studies, is approximately $2 \mathrm{~mm} .{ }^{11} \mathrm{~A}$ novel endovascular device consisting of a single-lumen, radiopaque markerlabeled, noncompliant segmental sealing, serial doubleballoon monorail catheter (Acrostak) was used for temporary, short-segment, parent artery occlusion with simultaneous use of the tip-side port as the shunting port maintaining the blood flow of the vessel, thus allowing the execution of a "dry" bypass surgery under recipient artery patency (Fig. 1).

Because we had no prior experience with the endovascular prototype device and were anticipating a significant learning curve, the study was designed in the following way: after conducting the experimental procedure on the first 4 animals, the device design was reevaluated and changes were made according to the initial experience. In addition to design changes of the device we also allowed for slight modifications of the perioperative protocol (e.g., medication) based on the experience in the first 4 animals.

All animals received postoperative supportive care, including a fentanyl patch $(12 \mu \mathrm{g} / \mathrm{hr})$ and meloxicam administered subcutaneously $(0.5 \mathrm{mg}$ /day $)$ for pain relief, and were closely monitored for any clinical (especially neurological) dysfunction. Systemic drugs to prevent the development of vasospasm were not given. A correlating MRI study was intended for all animals, including diffusion-weighted imaging (DWI) and 3D time-of-flight angiography (TOF-A) sequences in the postoperative period.

The following outcome measures were obtained and noted for each animal: patency of bypass based on visual inspection, presence of vasospasm on intraoperative selective left-sided carotid angiogram, surgical and endovascular complications, operating time, postoperative neurolog- ical condition, postoperative infarcts on MRI/DWI, and bypass patency on MRI TOF-A sequences.

After induction of general anesthesia under continuous cardiovascular monitoring (invasive blood pressure measurement, heart rate, oxygen saturation; see Appendix 1) by a certified team of veterinarians, the animal was positioned supine, and the skin over the neck and inguinal region was shaved and disinfected. The following surgical steps were performed under an operating microscope. First, the right femoral artery (FA) was surgically exposed under an operating microscope to allow for the insertion of a continuous normal saline-flushed introducer $(4-\mathrm{Fr}, 7-\mathrm{cm}$ percutaneous sheath; Arrow International). Next a cervical midline incision was performed and both common carotid arteries (CCAs) were exposed (Fig. 2A). The right CA was occluded with a temporary clip (Yaşargil Miniclip), transected proximally, and the vessel stump was flushed with heparinized saline and mobilized from the transection distally. In order to perform an end-to-side anastomosis, the right CA was transposed toward the left CA to perform a tension-free anastomosis at a $90^{\circ}$ angle. Next the left CA was exposed circumferentially at the level of the desired anastomosis and a blue rubber band was placed underneath the vessel (Fig. 2B). Details of this technique have been described in previous studies by our group. ${ }^{12,13}$

Under high-definition fluoroscopy with a single-plane C-arm digital subtraction angiography (DSA) machine (BV Pulsera, Philips Medical Systems), left-sided access of the left proximal CCA with a continuous normal saline-flushed microcatheter (1.7-Fr, 150-cm Excelsior SL10 straight microcatheter, Stryker; and 0.014-inch, 200$\mathrm{cm}$ Synchro microwire, ${ }^{14}$ Stryker) was subsequently performed using the over-the-wire technique. DSA of the left CCA and ICA was acquired at 2 frames per second in the posteroanterior view by injection of an iodinated contrast agent (3 mL, Iopamiro 300; Bracco Suisse SA) for exclusion of relevant vasoconstriction, parent artery thrombosis, or anastomotic leakage. Thereafter, right-sided access to the left ICA with a microwire (0.014-inch, 200-cm Synchro; ${ }^{14}$ Stryker) was performed using remask roadmap images for support guidance for subsequent radiopaque marker-labeled device placement in monorail technique covering the planned arteriotomy site with confirmatory angiography of the device localization, as well as exclusion of relevant vasoconstriction or recipient artery occlusion. Finally, serial double-balloon monorail catheter inflation over the anastomosis under fluoroscopic and external control, followed by confirmatory angiographic flow assessment through the device shunting port after microwire removal was carried out.

After revisiting the results and experiences of animal experiments $1-4$, a change in protocol was implemented for animal experiments 5-10, including bilateral insertion of continuously saline-flushed introducers $(4-\mathrm{Fr}, 7-\mathrm{cm}$ percutaneous sheath; Arrow International) providing simultaneous access to the left CCA with a continuously flushed microcatheter under thrombosis and vasospasm prophylaxis (saline infusion at $18 \mathrm{ml} / \mathrm{hr}$ including $50 \mathrm{IU}$ heparin $/ \mathrm{kg}$ of body weight and $0.05 \mathrm{mg}$ nimodipine $/ \mathrm{kg}$ of body weight), as well as with the device. Also, devicespecific adaptations, including shortening of the distal tip 

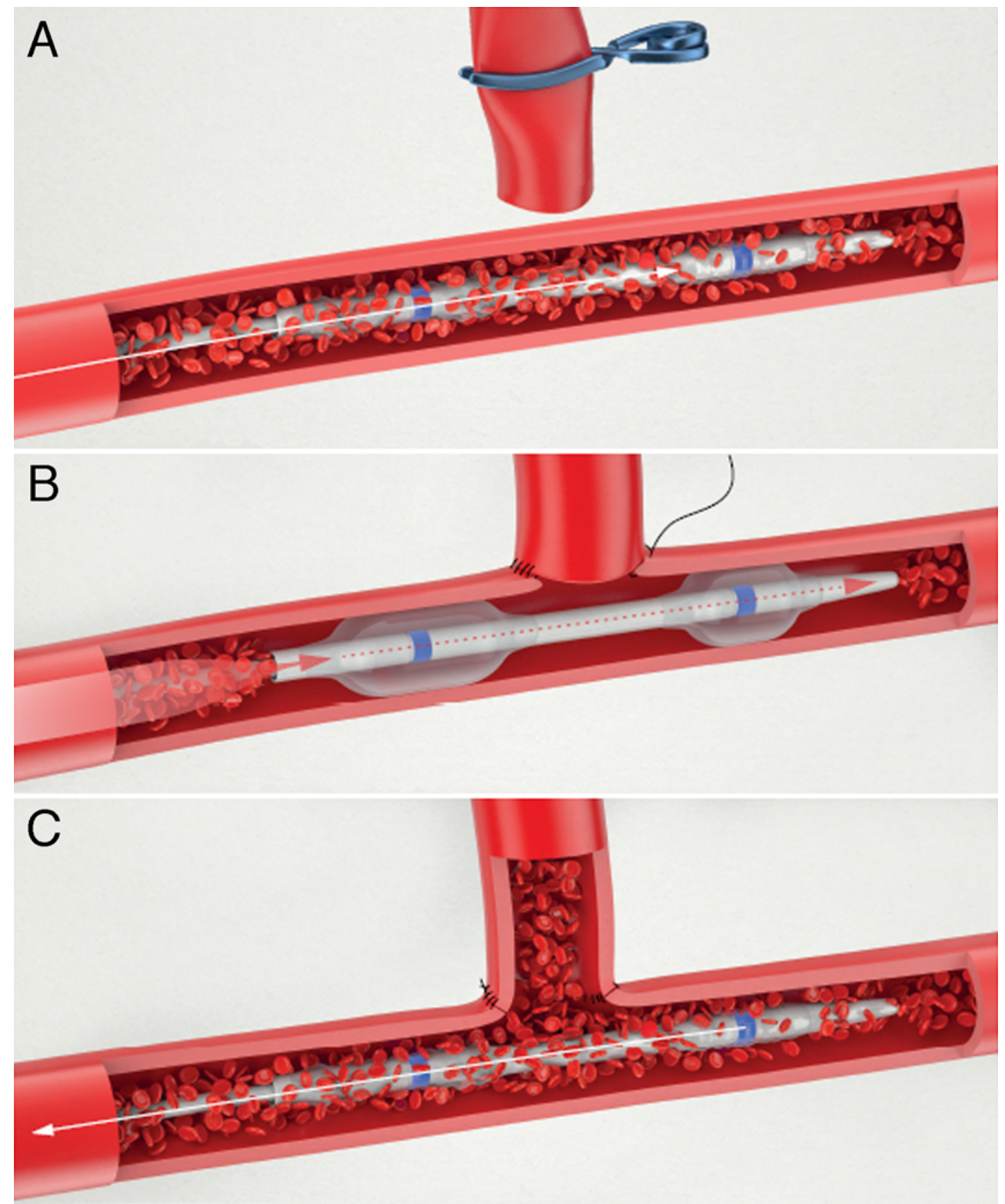

FIG. 1. A: Deflated endovascular device directed into the parent vessel. B: Inflated endovascular device in position while central parent vessel flow is maintained. C: After completion of the bypass the device is deflated and removed. Copyright Ruth Angliker. Published with permission. Figure is available in color online only.

and overall length, smaller balloon diameter, distance and postdeflation profile reduction, silicon balloon coating, and radiopaque marker labeling, were performed.

As a last step, an arteriotomy was performed in the left $\mathrm{CA}$ at the level of the inflated endovascular device (Fig. 2C). In the usual fashion, the right CA stump was anastomosed onto the left carotid arteriotomy by using an interrupted suture technique with 10-0 Dafilon monofilament. At this point the endovascular device was deflated slowly to allow for inspection of the anastomosis and, if necessary, placement of additional sutures (Fig. 2D). Once no significant leakage was noted, the device and temporary clip were removed from the distal right CA and a selective left CCA angiogram was performed. The angiogram was checked for patency of the anastomosis, signs of vasospasm, or leakage from the anastomosis.

At this point the foreign material was removed and the incision sites were closed in a layered fashion. The 

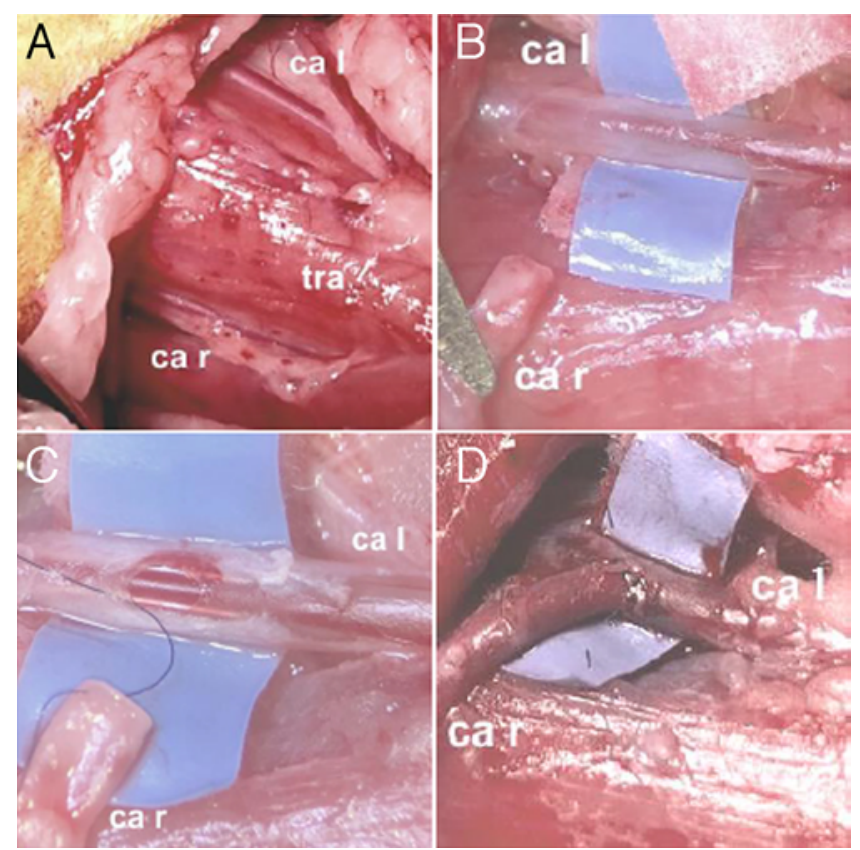

FIG. 2. A: Exposure of the cervical region. B: Preparation for end-toside anastomosis of the right-to-left CA. C: "Dry" arteriotomy of the left CA using the ENO technique. D: Patent bypass. ca I = CA left; ca $r=C A$ right; tra $=$ trachea. Figure is available in color online only.

animals' clinical condition, especially gross neurological function, was closely observed in the postoperative period in a standardized way by using the Animal Health Score Sheet on days 1, 2, and 3 (at 1 Am, 6 AM, 2 PM, 6 PM, and $10 \mathrm{PM})$. Occurrences of severe neurological deficits, airway obstruction, or circulatory compromise led to euthanasia of the animals, per protocol.

In the early postoperative period the animals were transferred to a 1.5-T clinical MRI suite (Magnetom Avanto Syngo B17; Siemens Medical Solutions), where sedation with an intravenous bolus injection of ketamine was established while the animals were spontaneously breathing in the supine position with oxygen augmentation via cannula. MRI was performed after manual bolus injection of GadovistH $(0.1 \mathrm{ml} / \mathrm{kg}$ Gadubutrol; Schering AG), including the following sequences: T2 space; DWI; T1 fat suppression; TOF; 3D angiography; and T1 magnetization-prepared rapid acquisition gradient echo (MPRAGE; field of view $137-200 \mathrm{~mm}, 1-\mathrm{mm}$ section thickness, matrix $352 \times 512$ ). Morphometric measurement of 3D vessel reconstructions was performed using the Siemens workstation (Fig. 3). Further details on the imaging protocols is given in a previous study by our group..$^{12}$

Following the examination, all animals were euthanized by applying an intravenous bolus of phenobarbital. The primary endpoint was defined as patency of the bypass at follow-up. Secondary endpoint studies included occurrence of vasospasm, neurological deficits, and operating time.

\section{Results}

This study showed the technical feasibility of the ENO

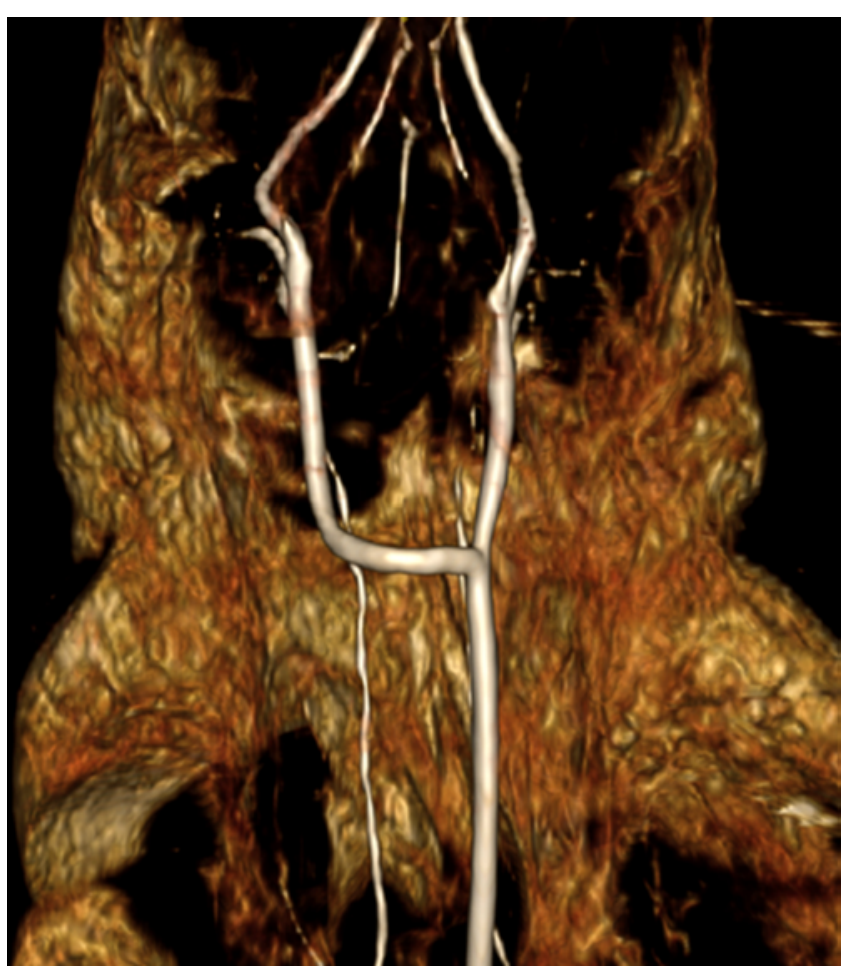

FIG. 3. 3D reconstructed MRA of the cervical vessels showing a patent right-to-left CA bypass with no signs of thrombosis or vasospasm. Figure is available in color online only.

high-flow bypass in a rabbit model. A bypass was successfully created in all animals. Positioning of the endovascular device was possible in all animals and the anastomosis could be sutured without any complication. No problems occurred while deflating and retrieving the ENO device. Final intraoperative DSA showed a patent bypass in 10 of 10 animals. The bypass remained patent in $2(50 \%)$ of 4 animals on postoperative MRA control imaging, and none of the animals demonstrated ischemic lesions. A detailed listing of all obtained parameters for each animal is given in Table 1. The mean operating time was 275 minutes (SD 75 minutes). Two major procedural complications were noted. A CA perforation in animal 1 led to a change of design and was not encountered again thereafter. Delayed FA hemorrhage in animal 5, which led to euthanasia, was due to a surgical error. The cause for a severe postoperative stridor in animal 8 , which also led to euthanasia of the animal, remained unresolved. None of the animals unaffected by these 3 complications showed any postoperative deficits indicative of an ischemic event.

\section{Discussion}

Development of new therapies requires animal models with well-defined extra- and intracranial vasculature. The rabbit as a small-animal model has many desirable traits, and with improved DSA and small-catheter techniques allows good anatomical definition.

We showed the feasibility of a novel technique to estab- 
TABLE 1. Results of ENO high-flow bypass in 10 rabbits

\begin{tabular}{ccccc}
\hline $\begin{array}{c}\text { Animal } \\
\text { No. }\end{array}$ & Bypass Patency Intraop & Complications & Vasospasm & Postop Neurological Condition \\
\hline 1 & Yes & CA perforation & None & NA \\
\hline 2 & Yes & None & Mild & Good \\
\hline 3 & Yes & None & None & NA \\
\hline 4 & Yes & None & Severe & Good \\
\hline 5 & Yes & Delayed FA hemorrhage & Mild & NA \\
\hline 6 & Yes & None & None & Good \\
\hline 7 & Yes & None & None & Good \\
\hline 8 & Yes & Severe stridor & None & NA \\
\hline 9 & Yes & None & None & Good \\
\hline 10 & Yes & None & None & Good \\
\hline NA $=$ not applicable & & & &
\end{tabular}

lish a high-flow cerebral bypass without parent vessel occlusion by using an endovascular device, which partially occluded the parent vessel at the site of the anastomosis. Immediate bypass patency was achieved in all animals and none of the animals that underwent MRI showed ischemic sequelae on follow-up. In 2 animals operative complications occurred, and 2 animals experienced thrombosis of their bypass on follow-up MRA.

Despite the advantages of endovascular surgery and radiosurgery, there are still valuable indications for highflow bypass procedures. The advantages of our ENO technique are its nonocclusive nature and the fact that less space is required around the site of arteriotomy to perform the actual bypass as compared to the conventional technique, which requires temporary clips to be in place proximal and distal to the anastomosis. Surgical situations that could particularly benefit from these advantages are the Fukushima bypass ${ }^{9}$ and posterior circulation bypasses..$^{15-17}$ In both situations the ENO technique would facilitate the creation of an anastomosis because no temporary clips are necessary on the target vessel, which would further crowd the surgical field. Potential target vessels could be the first segment of the MCA (i.e., $\mathrm{M}_{1}$ ) or the second segment of the posterior cerebral artery (PCA; i.e., $\left.\mathrm{P}_{2}\right)$; each is on average $2.2 \mathrm{~mm}$ in diameter. ${ }^{18,19}$

As known from previous studies, temporary clipping of central cerebral vessels such as the $\mathrm{M}_{1}$ segment carries the risk of ischemia, especially when exceeding an occlusion time of 10-19 minutes. ${ }^{7,8}$ Certainly, the main advantage of our novel technique, the ENO bypass, is its nonocclusive nature, which reduces the risk of infarcts related to temporary vessel occlusion. Again this advantage could be of particular significance in the case of difficult, time-consuming anastomoses, such as posterior circulation bypasses, which can be indicated in the treatment of moyamoya disease, PCA aneurysms, complex basilar apex aneurysms, or dolichoectatic basilar artery aneurysms. ${ }^{14-17}$

Notably, vasospasm was observed in 3 of 10 animals on intraoperative selective carotid angiography in the left $\mathrm{CA}$ as well as the anastomosed right CA due to surgical and endovascular manipulation. We speculate that the occurrence of vasospasm might be a contributing factor to short-term bypass thrombosis and the rather low rate of bypass patency on follow-up MRI/MRA (50\%). Endothelial damage and subsequent thrombosis by the endovascular device might be another mechanism implicated in bypass thrombosis in our series. Future studies should examine both short- and long-term bypass patency and should implement strategies to prevent vasospasm, such as further design modification as well as topical or systemic application of calcium channel blockers and temporary induced hypertension. In addition, similar to protocols in patients who receive bypass surgery, systemic platelet inhibitors or heparin should be implemented to decrease the rate of short-term bypass thrombosis. Future studies have to investigate possible endothelial damage by the endovascular device. Given the unique properties of the endovascular device under investigation, further indications could be explored in future studies such as safety balloon for aneurysm coil placement in case of intraprocedural rupture.

Limitations of the study are as follows: the small number of procedures performed, and the lack of histological analysis of the bypass and parent vessel to confirm endothelial integrity. Furthermore, the study lacks a potent prophylactic regimen to reduce the occurrence of vasospasm and potentially bypass thrombosis. Because rabbits are known to have very robust collateral flow and their vasculature is prone to vasospasm, a different research animal might be chosen in the future to more realistically simulate the hemodynamic conditions in humans.

\section{Conclusions}

We have shown in principle the feasibility of bypass surgery without temporary vessel occlusion by using an endovascular nonocclusive device in a rabbit model. These preliminary results warrant further device optimization and refinement of the technique, including perioperative management to prevent vasospasm and improve short- and long-term patency. A larger series and long-term followup studies are needed to evaluate the efficacy and safety of this promising technique. 


\section{Acknowledgments}

This study has been financially supported by the Research Fund of the Department of Neurosurgery, Kantonsspital Aarau, Switzerland. We thank Erwin Berger of Acrostak Schweiz AG in Winterthur, Switzerland, for device development, and Olgica Beslac and Kay Nettelbeck of the Experimental Surgery Facility (ESF), Department for Biomedical Research, University of Bern, Switzerland, for their skillful management of animal care and operative assistance. We further express our gratitude to Ruth Angliker for illustrations.

\section{References}

1. Langer DJ, Vajkoczy P. ELANA: excimer laser-assisted nonocclusive anastomosis for extracranial-to-intracranial and intracranial-to-intracranial bypass: a review. Skull Base. 2005;15(3):191-205.

2. Liu JK, Couldwell WT. Interpositional carotid artery bypass strategies in the surgical management of aneurysms and tumors of the skull base. Neurosurg Focus. 2003;14(3):e2 .

3. Reynolds MR, Derdeyn CP, Grubb RL Jr, et al. Extracranialintracranial bypass for ischemic cerebrovascular disease: what have we learned from the Carotid Occlusion Surgery Study? Neurosurg Focus. 2014;36(1):E9.

4. Yaşargil MG, Yonekawa Y. Results of microsurgical extraintracranial arterial bypass in the treatment of cerebral ischemia. Neurosurgery. 1977;1(1):22-24.

5. Sia SF, Morgan MK. High flow extracranial-to-intracranial brain bypass surgery. J Clin Neurosci. 2013;20(1):1-5.

6. Nossek E, Langer DJ. Internal maxillary artery to middle cerebral artery cranial bypass: the new "work horse" for cerebral flow replacement. World Neurosurg. 2018;115:44-46.

7. Lavine SD, Masri LS, Levy ML, Giannotta SL. Temporary occlusion of the middle cerebral artery in intracranial aneurysm surgery: time limitation and advantage of brain protection. J Neurosurg. 1997;87(6):817-824.

8. Samson D, Batjer HH, Bowman G, et al. A clinical study of the parameters and effects of temporary arterial occlusion in the management of intracranial aneurysms. Neurosurgery. 1994;34(1):22-29.

9. Spetzler RF, Fukushima T, Martin N, Zabramski JM. Petrous carotid-to-intradural carotid saphenous vein graft for intracavernous giant aneurysm, tumor, and occlusive cerebrovascular disease. J Neurosurg. 1990;73(4):496-501.

10. Kilkenny C, Browne WJ, Cuthill IC, et al. Improving bioscience research reporting: the ARRIVE guidelines for reporting animal research. PLoS Biol. 2010;8(6):e1000412.

11. Lee JS, Hamilton MG, Zabramski JM. Variations in the anatomy of the rabbit cervical carotid artery. Stroke. 1994;25(2):501-503.

12. Marbacher S, Erhardt S, Schläppi JA, et al. Complex bilobular, bisaccular, and broad-neck microsurgical aneurysm formation in the rabbit bifurcation model for the study of upcoming endovascular techniques. AJNR Am J Neuroradiol. 2011;32(4):772-777.

13. Sherif C, Marbacher S, Erhardt S, Fandino J. Improved microsurgical creation of venous pouch arterial bifurcation aneurysms in rabbits. AJNR Am J Neuroradiol. 2011;32(1):165169.
14. Kimiwada T, Hayashi T, Shirane R, Tominaga T. Posterior cerebral artery stenosis and posterior circulation revascularization surgery in pediatric patients with moyamoya disease. J Neurosurg Pediatr. 2018;21(6):632-638.

15. Lawton MT, Abla AA, Rutledge WC, et al. Bypass surgery for the treatment of dolichoectatic basilar trunk aneurysms: a work in progress. Neurosurgery. 2016;79(1):83-99.

16. Liu Y, Shi X, Kc KIS, et al. Microsurgical treatment for complex basilar artery aneurysms with long-term follow-up in a series of 35 cases. World Neurosurg. 2018;111:e710-e721.

17. Zador Z, Lu DC, Arnold CM, Lawton MT. Deep bypasses to the distal posterior circulation: anatomical and clinical comparison of pretemporal and subtemporal approaches. Neurosurgery. 2010;66(1):92-101.

18. Uz A. The segmentation of the posterior cerebral artery: a microsurgical anatomic study. Neurosurg Rev. 2019;42(1):155-161.

19. Zurada A, Gielecki J, Tubbs RS, et al. Three-dimensional morphometrical analysis of the M1 segment of the middle cerebral artery: potential clinical and neurosurgical implications. Clin Anat. 2011;24(1):34-46.

\section{Disclosures}

The authors report no conflict of interest concerning the materials or methods used in this study or the findings specified in this paper.

\section{Author Contributions}

Conception and design: Spiessberger, Gruter, Diepers, Remonda, Añon. Acquisition of data: Strange, Gruter, Wanderer, Casoni, Diepers, Remonda, Añon. Analysis and interpretation of data: Spiessberger, Strange. Drafting the article: Spiessberger, Casoni. Critically revising the article: all authors. Approved the final version of the manuscript on behalf of all authors: Spiessberger. Administrative/technical/material support: Strange, Gruter, Wanderer, Casoni, Diepers, Remonda, Añon. Study supervision: Fandino, Añon, Marbacher.

\section{Supplemental Information \\ Online-Only Content}

Supplemental material is available with the online version of the article.

Appendix 1. https://thejns.org/doi/suppl/10.3171/2020.3. JNS20334.

\section{Previous Presentations}

The study was orally presented at the AANS Annual Scientific Meeting in San Diego, CA, on April 16, 2019; the EANS Vascular Meeting in Nice, France, on September 5-6, 2019; and the Bypass 2020 Anniversary Symposium in Zurich, Switzerland, on January 9,2020

\section{Correspondence}

Alexander Spiessberger: c/o Neuro Research Office, Kantonsspital Aarau, Switzerland. aspiessberger@gmx.ch; neurosurgery@ksa.ch. 